ANNALES

POLONICI MATHEMATICI

LXXVII.3 (2001)

\title{
Invariance of domain in o-minimal structures
}

\author{
by RafaŁ PierzchaŁa (Kraków)
}

\begin{abstract}
The aim of this paper is to prove the theorem on invariance of domain in an arbitrary o-minimal structure. We do not make use of the methods of algebraic topology and the proof is based merely on some basic facts about cells and cell decompositions.
\end{abstract}

1. Preliminaries. For the convenience of the reader we recall some notions and facts on o-minimal structures (cf. [1]).

(1.1) A linearly ordered set $R$ is called dense if for all $a, b \in R$ with $a<b$ there is $c \in R$ such that $a<c<b$. Let $(R,<)$ be a dense linearly ordered set without endpoints, that is, $R$ has no largest or smallest element. We add two endpoints $-\infty,+\infty$ satisfying $-\infty<a<+\infty$ for all $a \in R$ and define open and closed intervals respectively by

$$
\begin{aligned}
& (a, b)=\{x \in R \mid a<x<b\} \quad \text { where }-\infty \leq a<b \leq+\infty, \\
& {[a, b]=\{x \in R \mid a \leq x \leq b\} \quad \text { where }-\infty<a<b<+\infty .}
\end{aligned}
$$

We take $R$ equipped with the order topology. By an open box in $R^{n}$ we will mean the cartesian product of $n$ open intervals. Then open boxes form the base of a topology in $R^{n}$.

$(1.2)$ Let $(R,<)$ be a dense linearly ordered nonempty set without endpoints.

Definition. An o-minimal structure on $R$ is a sequence $\mathcal{S}=\left(\mathcal{S}_{n}\right)_{n \in \mathbb{N}}$ such that for each $n$ :

1. $\mathcal{S}_{n}$ is a boolean algebra of subsets of $R^{n}$;

2. If $A \in \mathcal{S}_{n}$, then $A \times R$ and $R \times A$ belong to $\mathcal{S}_{n+1}$;

3. $\left\{\left(x_{1}, \ldots, x_{n}\right) \in R^{n} \mid x_{1}=x_{n}\right\} \in \mathcal{S}_{n}$;

4. If $A \in \mathcal{S}_{n+1}$, then $\pi(A) \in \mathcal{S}_{n}$, where $\pi: R^{n+1} \rightarrow R^{n}$ is the projection on the first $n$ coordinates;

2000 Mathematics Subject Classification: Primary 32B20, 32K99; Secondary 14P10.

Key words and phrases: o-minimal structure, cell decomposition, definable set, stratification, invariance of domain. 
5. $\left\{(x, y) \in R^{2} \mid x<y\right\} \in \mathcal{S}_{2}$

6 . The sets in $\mathcal{S}_{1}$ are exactly finite unions of intervals and points.

(1.3) We shall now recall an example of an o-minimal structure on the ordered set $\mathbb{R}$ of real numbers. A semialgebraic subset of $\mathbb{R}^{n}$ is a subset defined by a finite system of polynomial equations and inequalities with real coefficients. By the Tarski-Seidenberg theorem the semialgebraic sets satisfy axiom 4 in the definition above. The remaining axioms are obviously fulfilled, so the semialgebraic sets form an o-minimal structure.

(1.4) From now on we fix an o-minimal structure $\mathcal{S}$ on $R$. Let $A \subset R^{n}$ and $f: A \rightarrow R^{m}$. We say $A$ is definable if $A \in \mathcal{S}_{n}$. We say the map $f$ is definable if its graph $\Gamma(f) \subset R^{n+m}$ is definable. If $f$ is definable, then the domain $A$ of $f$ and its image $f(A)$ are also definable. Moreover, if $f$ is injective, its inverse $f^{-1}$ is definable.

(1.5) If $A \subset R^{n}$ is definable, so are its closure, interior, boundary and frontier, where the frontier of $A$ is $\partial A=\bar{A} \backslash A$.

(1.6) A set $X \subset R^{n}$ is called definably connected if $X$ is definable and $X$ is not the union of two disjoint nonempty definable open subsets of $X$. Of course, an interval is definably connected. A definably connected component of a definable set $X \subset R^{n}$ is, by definition, a maximal definably connected subset of $X$.

(1.7) For each definable set $X \subset R^{n}$ we put

$$
C(X)=\{f: X \rightarrow R \mid f \text { is definable and continuous }\} .
$$

Let $C_{\infty}(X)=C(X) \cup\{-\infty,+\infty\}$, where we regard $-\infty$ and $+\infty$ as constant functions on $X$. For $f, g$ in $C_{\infty}(X)$ we write $f<g$ if $f(x)<g(x)$ for all $x \in X$, and in this case we put

$$
(f, g)=\{(x, r) \in X \times R \mid f(x)<r<g(x)\} .
$$

(1.8) Definition. Let $\left(i_{1}, \ldots, i_{n}\right)$ be a sequence of zeros and ones of length $n$. An $\left(i_{1}, \ldots, i_{n}\right)$-cell is a definable subset of $R^{n}$ obtained by induction on $n$ as follows:

- a (0)-cell is a point $\{r\} \subset R$, a (1)-cell is an interval $(a, b) \subset R$;

- suppose $\left(i_{1}, \ldots, i_{n}\right)$-cells are already defined; then an $\left(i_{1}, \ldots, i_{n}, 0\right)$-cell is the graph $\Gamma(f)$ of a function $f \in C(X)$, where $X$ is an $\left(i_{1}, \ldots, i_{n}\right)$-cell; further, an $\left(i_{1}, \ldots, i_{n}, 1\right)$-cell is a set $(f, g)$, where $f, g \in C_{\infty}(X), f<g$ and $X$ is an $\left(i_{1}, \ldots, i_{n}\right)$-cell.

A cell in $R^{n}$ is an $\left(i_{1}, \ldots, i_{n}\right)$-cell for some sequence $\left(i_{1}, \ldots, i_{n}\right)$. We call $(1, \ldots, 1)$-cells open cells.

(1.9) Definition. A cell decomposition of $R^{n}$ is a partition of $R^{n}$ into finitely many cells defined inductively as follows: 
- a cell decomposition of $R^{1}=R$ is a collection

$$
\left\{\left(-\infty, a_{1}\right),\left(a_{1}, a_{2}\right), \ldots,\left(a_{k},+\infty\right),\left\{a_{1}\right\}, \ldots,\left\{a_{k}\right\}\right\}
$$

where $a_{1}<\ldots<a_{k}$ are points in $R$;

- a cell decomposition of $R^{n+1}$ is a finite partition of $R^{n}$ into cells $A$ such that the set of projections $\pi(A)$ is a cell decomposition of $R^{n}$.

(1.10) Cell Decomposition Theorem [1]. Given a finite collection of definable sets in $R^{n}$ there is a cell decomposition of $R^{n}$ partitioning each of them.

(1.11) We define the dimension of a nonempty definable set $X \subset R^{n}$ by $\operatorname{dim} X=\max \left\{i_{1}+\ldots+i_{n} \mid X\right.$ contains an $\left(i_{1}, \ldots, i_{n}\right)$-cell $\}$.

To the empty set we assign the dimension $-\infty$.

A nonempty definable set $X \subset R^{n}$ is called purely $k$-dimensional if for each nonempty definable open subset $U \subset X$ we have $\operatorname{dim} U=\operatorname{dim} X=k$.

(1.12) Theorem [1]. Let $X$ be a nonempty definable subset of $R^{n}$. Then $\operatorname{dim} \partial X<\operatorname{dim} X$.

(1.13) A stratification of $R^{n}$ is a partition $\mathcal{T}$ of $R^{n}$ into finitely many cells such that for each cell $A \in \mathcal{T}, \partial A$ is a union of cells of $\mathcal{T}$.

(1.14) Theorem [1]. Given any definable sets $A_{1}, \ldots, A_{k} \subset R^{n}$ there is a stratification of $R^{n}$ partitioning each of $A_{1}, \ldots, A_{k}$.

(1.15) TheOrem [2]. If $f: X \rightarrow R^{n}$ is a definable map on a closed bounded set $X \subset R^{m}$, then $f(X)$ is closed and bounded in $R^{n}$.

(1.16) THEOREM [1]. If $X \subset R^{n}, Y \subset R^{m}$ are definable and there is a definable bijection between $X$ and $Y$, then $\operatorname{dim} X=\operatorname{dim} Y$.

(1.17) Corollary. Let $f: A_{1} \rightarrow A_{2}$ be a definable continuous bijection, where $A_{1}$ is a purely $k$-dimensional subset of $R^{n}$ and $A_{2} \subset R^{m}$. Then $A_{2}$ is a purely $k$-dimensional subset of $R^{m}$.

2. Some properties of cells. In this section we prove some lemmas on cells. In the proofs we assume that if a cell $\theta$ is of the form $(f, g)$, then $f, g \in C(\pi(\theta))$. We do not consider cells of the form $(-\infty, f),(f,+\infty)$, $X \times R$, because the corresponding proofs are simple modifications of the cases handled below.

(2.1) Lemma. If $G$ is an open cell in $R^{n}$, then $\operatorname{int} \bar{G}=G$.

Proof. The lemma is clear for $n=1$. Let $n>1$ and assume inductively the lemma holds for $n-1$. Take $G=(f, g)$ with $f, g \in C(\Omega)$, where $\Omega$ is an open cell in $R^{n-1}$. We have $\bar{G}=[\bar{G} \cap((\bar{\Omega} \backslash \Omega) \times R)] \cup G \cup \Gamma(f) \cup \Gamma(g)$. If $x \in \operatorname{int} \bar{G}$, then $x \in W \subset \bar{G}$, where $W$ is an open set in $R^{n}$. Clearly, $\pi(W)$ 
is open in $R^{n-1}$ and $\pi(x) \in \pi(W) \subset \bar{\Omega}$, so $\pi(x) \in \operatorname{int} \bar{\Omega}$. By the induction hypothesis $\pi(x) \in \Omega$. Therefore $x \in G$ and int $\bar{G} \subset G$. The reverse inclusion is trivial.

(2.2) Lemma. Let $\theta \subset R^{n}$ be a cell with $\operatorname{dim} \theta=n-1$ and let $G$ be an open cell such that $\theta \subset \partial G$. Then there is a definable set $Z \subset \theta$ such that $\operatorname{dim} Z<n-1$ and for each $a \in \theta \backslash Z$ there exist arbitrarily small open boxes $K$ satisfying the following condition: $a \in K$ and $K \backslash \theta$ has exactly two definably connected components $K_{1}, K_{2}$, both open in $R^{n}$ and $K_{1} \subset G$, $K_{2} \cap G=\emptyset$.

Proof. By induction on $n$. The case $n=1$ is obvious. Suppose $n>1$ and that the lemma holds for $n-1$.

CASE 1: The cell $\theta$ is of the form $(\varphi, \psi)$ with $\varphi, \psi \in C(\pi(\theta))$. One checks easily that $\pi(\theta) \subset \partial \pi(G)$. By the induction hypothesis applied to the cells $\pi(\theta)$ and $\pi(G)$ there is a definable set $Z^{\prime} \subset \pi(\theta)$ satisfying the required conditions. We put $Z=\theta \cap\left[\overline{\Gamma(f)} \cup \overline{\Gamma(g)} \cup\left(Z^{\prime} \times R\right)\right]$ with $f, g \in C(\pi(G))$ such that $G=(f, g)$. Clearly, $Z=\theta \cap\left[\partial \Gamma(f) \cup \partial \Gamma(g) \cup\left(Z^{\prime} \times R\right)\right]$, so $\operatorname{dim} Z<n-1$. Let $a \in \theta \backslash Z$ and let $L$ be an open box such that $a \in L$ and $L \cap[\Gamma(f) \cup \Gamma(g)]=\emptyset$. We may assume that $L$ is arbitrarily small and $\pi(L \backslash \theta) \cap \pi(\theta)=\emptyset$ (replacing $L$ by a smaller box, if necessary). We have $\pi(a) \in \pi(\theta) \backslash Z^{\prime}$, so there is an open box $K^{\prime}$ in $R^{n-1}$ such that $\pi(a) \in K^{\prime}$ and $K^{\prime} \backslash \pi(\theta)$ has exactly two definably connected components $K_{1}^{\prime}, K_{2}^{\prime}$, both open in $R^{n-1}$ and $K_{1}^{\prime} \subset \pi(G), K_{2}^{\prime} \cap \pi(G)=\emptyset$. Since $K^{\prime}$ can be chosen arbitrarily small, we may assume that $K^{\prime} \subset \pi(L)$. We put $K=K^{\prime} \times I$, $K_{1}=K_{1}^{\prime} \times I, K_{2}=K_{2}^{\prime} \times I$, where $I$ is an interval such that $L=\pi(L) \times I$. One easily verifies that the box $K$ has the required property.

Case 2: The cell $\theta$ is of the form $\Gamma(\varphi), \varphi \in C(\pi(\theta))$. We have $G=$ $(f, g)$ with $f, g \in C(\pi(G))$ and $\pi(\theta), \pi(G)$ are open cells in $R^{n-1}$. Note that $\pi(\theta) \subset \operatorname{int} \overline{\pi(G)}$. Hence by $(2.1), \pi(\theta) \subset \pi(G)$ and either $\varphi=f \mid \pi(\theta)$ or $\varphi=g \mid \pi(\theta)$. The rest of the proof is now straightforward. In this case we can take $Z=\emptyset$.

(2.3) Remark. In general, we cannot expect to have $Z=\emptyset$ in the previous lemma. Consider a semialgebraic map $f: \mathbb{R} \times(0,+\infty) \rightarrow \mathbb{R}$ such that $\Gamma(f)=\Gamma\left(g_{1}\right) \cup \Gamma\left(g_{2}\right)$ with

$$
\begin{aligned}
& g_{1}:[0,+\infty) \times(0,+\infty) \ni(x, y) \mapsto x / y \in \mathbb{R}, \\
& g_{2}:(-\infty, 0] \times(0,+\infty) \ni(x, y) \mapsto-x / y \in \mathbb{R} .
\end{aligned}
$$

We put $G=(-\infty, f)$ and $\theta=\mathbb{R} \times\{0\} \times \mathbb{R}$.

(2.4) Lemma. Let $G$ and $H$ be disjoint open cells in $R^{n}$ and let $\theta$ be a cell of dimension $n-1$ contained in $\partial G \cap \partial H$. Then there is a definable 
set $Z \subset \theta$ such that $\operatorname{dim} Z<n-1$ and $(\theta \backslash Z) \cup G \cup H$ is open in $R^{n}$. In particular $\theta \backslash Z \subset \operatorname{int} \overline{G \cup H}$.

Proof. Applying Lemma (2.2) twice: to the cells $\theta, G$ and then to $\theta, H$, we obtain definable sets $Z_{1}, Z_{2}$. We put $Z=\theta \cap\left(\overline{Z_{1} \cup Z_{2}}\right)$. One checks easily that the set $Z$ satisfies the requirements.

(2.5) Lemma. Let $T$ be an open cell in $R^{n}$ and let $\theta$ be a cell of dimension $n-1$ contained in $\partial T$. Then there are no disjoint open cells $G, H$ contained in $T$ such that $\theta \subset \partial G \cap \partial H$.

Proof. Suppose there are disjoint open cells $G, H$ contained in $T$ such that $\theta \subset \partial G \cap \partial H$. By (2.4), $\theta \cap \operatorname{int} \overline{G \cup H} \neq \emptyset$ and hence $\theta \cap \operatorname{int} \bar{T} \neq \emptyset$. This is a contradiction, because int $\bar{T}=T$.

(2.6) Lemma. Let $V$ be an open box in $R^{n}$ and let $\Omega \subset V$ be an open definable set which is not definably connected. Suppose $\mathcal{C}$ is a cell decomposition of $R^{n}$ partitioning $V$ and $\Omega$. Then there is a cell $\theta \in \mathcal{C}$ of dimension $n-1$ such that $\theta \subset V \backslash \Omega$.

Proof. By induction on $n$, the case $n=1$ being trivial. Suppose the lemma holds for $n-1$, where $n>1$. We have $V=V^{\prime} \times I$ with $V^{\prime}$ a box in $R^{n-1}$ and $I$ an interval. By the assumption $\Omega=\Omega_{1} \cup \Omega_{2}$ with $\Omega_{1}, \Omega_{2}$ nonempty definable open subsets of $R^{n}$ such that $\Omega_{1} \cap \Omega_{2}=\emptyset$.

CASE 1: $\pi\left(\Omega_{1}\right) \cap \pi\left(\Omega_{2}\right) \neq \emptyset$. There is a cell $\theta^{\prime} \in \mathcal{C}^{\prime}$ of dimension $n-1$ such that $\theta^{\prime} \subset \pi\left(\Omega_{1}\right) \cap \pi\left(\Omega_{2}\right)$, where $\mathcal{C}^{\prime}$ denotes the corresponding cell decomposition of $R^{n-1}$. Clearly, for some cell $\theta \in \mathcal{C}$ of dimension $n-1$ we have $\theta \subset V \backslash \Omega$.

CASE 2: $\pi\left(\Omega_{1}\right) \cap \pi\left(\Omega_{2}\right)=\emptyset$. Applying the induction hypothesis to $V^{\prime}$ and $\pi(\Omega)$ we get a cell $\theta^{\prime} \subset V^{\prime}$ of dimension $n-2$ such that $\theta^{\prime} \cap \pi(\Omega)=\emptyset$. There is a cell $\theta \in \mathcal{C}$ of dimension $n-1$ such that $\pi(\theta)=\theta^{\prime}$ and $\theta \subset V$. Obviously, $\theta \cap \Omega=\emptyset$.

\section{Main result}

THEOREM. Let $\Omega_{1}$ be an open definable subset of $R^{n}$ and let $f: \Omega_{1} \rightarrow \Omega_{2}$ be a definable homeomorphism onto a definable set $\Omega_{2} \subset R^{n}$. Then $\Omega_{2}$ is open in $R^{n}$.

Proof. Given $a \in \Omega_{1}$ we have to show that $b=f(a) \in \operatorname{int} \Omega_{2}$. Let $K$ be a closed box in $R^{n}$ (i.e. a cartesian product of $n$ closed intervals) such that $a \in \operatorname{int} K$ and $K \subset \Omega_{1}$. Note first that by (1.15), $f(K)$ is closed and bounded in $R^{n}$.

We take a stratification $\mathcal{C}$ of $R^{n}$ that partitions $f(K)$. Let $\mathcal{A}$ be a collection of all open cells $A \in \mathcal{C}$ such that $b \in \bar{A}$. We define $\mathcal{F}=\{A \in \mathcal{A} \mid$ 
$A \subset f(K)\}$. Since $f(K)$ is a purely $n$-dimensional set (cf. (1.17)), it follows that $\mathcal{F} \neq \emptyset$. Suppose now that $b \notin \operatorname{int} f(K)$. Note that then $\mathcal{A} \backslash \mathcal{F} \neq \emptyset$.

Claim. There exist $G \in \mathcal{F}, H \in \mathcal{A} \backslash \mathcal{F}$ and a cell $\theta \in \mathcal{C}$ of dimension $n-1$ such that $b \in \bar{\theta}$ and $\theta \subset \partial G \cap \partial H$.

To see this, let $V$ be an open box containing $b$ and disjoint from any cell $A \in \mathcal{C}$ such that $b \notin \bar{A}$. We put $V_{1}=\bigcup\{A \mid A \in \mathcal{F}\}, V_{2}=\bigcup\{A \mid A \in \mathcal{A} \backslash \mathcal{F}\}$. By (2.6), $\operatorname{dim}(V \backslash \Omega)=n-1$, where $\Omega=V \cap\left(\operatorname{int} \bar{V}_{1} \cup \operatorname{int} \bar{V}_{2}\right)$. Let $\theta \in \mathcal{C}$ be a cell such that $\operatorname{dim}[(V \backslash \Omega) \cap \theta]=n-1$. Suppose the cell $\theta$ does not have the required property. Then $\theta \subset \partial G_{1} \cap \partial G_{2}$, where $G_{1} \neq G_{2}$ and $G_{1}, G_{2} \in \mathcal{F}$ or $G_{1}, G_{2} \in \mathcal{A} \backslash \mathcal{F}$. By Lemma $(2.4), \operatorname{dim}[(V \backslash \Omega) \cap \theta]<n-1$ and this is a contradiction.

Using (2.4) one easily checks that $\theta \cap \partial A=\emptyset$ whenever $A \in \mathcal{F}$ and $A \neq G$. Let $\mathcal{C}^{\prime}$ be a stratification of $R^{n}$ partitioning $f^{-1}(A)$ for all $A \in \mathcal{C}$. So $f^{-1}(\theta)$ is a purely $(n-1)$-dimensional set (cf. (1.17)), hence there is a cell $\theta^{\prime} \in \mathcal{C}^{\prime}$ of dimension $n-1$ such that $a \in \overline{\theta^{\prime}}$ and $\theta^{\prime} \subset f^{-1}(\theta)$. Clearly, $\theta^{\prime} \subset \partial G^{\prime}$ for some open cell $G^{\prime} \subset f^{-1}(G)$. Now we use the fact that $a \in \operatorname{int} K$ to conclude that there is an open cell $B \in \mathcal{C}^{\prime}$ such that $B \neq G^{\prime}, B \subset K$ and $\theta^{\prime} \subset \partial B$ (cf. (2.2)). It is routine to check that $B \subset f^{-1}(G)$. Taking a stratification of $R^{n}$ partitioning $f\left(\theta^{\prime}\right), f\left(G^{\prime}\right), f(B)$ we obtain a contradiction with Lemma (2.5).

Acknowledgements. I would like to thank Professor Wiesław Pawłucki for suggesting the problem and help during the preparation of this paper.

\section{References}

[1] L. van den Dries, Tame Topology and o-Minimal Structures, Cambridge Univ. Press, 1998.

[2] Y. Peterzil and C. Steinhorn, Definable compactness and definable subgroups of o-minimal groups, J. London Math. Soc. (2) 59 (1999), 769-786.

Institute of Mathematics

Jagiellonian University

Reymonta 4

30-059 Kraków, Poland

E-mail: rafal.pierzchala@im.uj.edu.pl 